\title{
La littérature comme expérience personnelle : la Macédoine et Stratis Myrivilis
}

\section{Georges Kostakiotis}

\section{OpenEdition}

1 Journals

Édition électronique

URL : https://journals.openedition.org/ceb/821

DOI : $10.4000 /$ ceb.821

ISSN : 2261-4184

Éditeur

INALCO

Édition imprimée

Date de publication : 30 mars 2011

Pagination : 167-180

ISBN : 978-2-85831-189-7

ISSN : 0290-7402

Référence électronique

Georges Kostakiotis, "La littérature comme expérience personnelle : la Macédoine et Stratis

Myrivilis », Cahiers balkaniques [En ligne], 38-39 | 2011, mis en ligne le 05 décembre 2011, consulté le

06 juillet 2021. URL : http://journals.openedition.org/ceb/821 ; DOI : https://doi.org/10.4000/ceb.821

Ce document a été généré automatiquement le 6 juillet 2021.

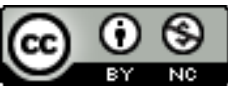

Cahiers balkaniques est mis à disposition selon les termes de la Licence Creative Commons Attribution - Pas d'Utilisation Commerciale 4.0 International. 


\title{
La littérature comme expérience personnelle : la Macédoine et Stratis Myrivilis
}

\author{
Georges Kostakiotis
}

1 Sous l'influence de Kostis Palamas qui parvient à lier la tradition grecque et la production intellectuelle occidentale ${ }^{1}$ et celle de Yiannis Psicharis qui ouvre sans complexes la route à l'utilisation de la langue démotique, Myrivilis est certainement un des écrivains les plus importants du début du XXe siècle. Il incarnera le passage des années 1880 aux années $1930^{2}$. D'une part, s'inspirant de son quotidien, il ne fait qu'exprimer la cruauté du monde, le « présent » douloureux et insupportable : « la dure réalité de la vie qui nous entoure et qui nous blesse, nous épuise, nous fait mal» déclare-t-il en effet ${ }^{3}$; d'autre part il montre son attachement étroit avec la tradition dont il ne se séparera jamais ${ }^{4}$. La tradition deviendra son seul appui durant les années 1930 ce qui lui coûtera la réputation d'être proche du régime de Métaxas bien qu'il ne l'ait jamais soutenu réellement. En effet, Myrivilis étant mal intégré dans la société bourgeoise athénienne et déçu de la stagnation de la vie actuelle a eu du mal à envisager l'avenir ; il se réfugie dans le passé, tel qu'il a été conservé dans ses souvenirs d'enfant en mettant en avant les héros de sa première jeunesse, le passé glorieux du monde grec et les traditions ancestrales mêlées à la grandeur de la nature. Il a su ainsi garder la fraîcheur du régionalisme et mettre en valeur sa Lesbos natale ${ }^{5}$.

2 Myrivilis est donc né dans l'île de Lesbos en 1890 et est mort à Athènes en 1967. Dans ce travail, nous nous intéresserons surtout à sa relation avec la Macédoine qu'il a connue durant sa jeunesse, comme soldat d'abord pendant les guerres balkaniques de 1912-1913 puis lors de la Première Guerre mondiale entre 1917 et 1919 quand la Grèce de Venizélos s'est rangée aux côtés des Alliés après l'attaque de la Bulgarie.

Myrivilis lui-même, comme la majorité des habitants de l'île de Lesbos d'ailleurs, a suivi Venizélos et l'a soutenu non seulement dans ses luttes politiques contre le roi, mais aussi pour la libération de la Macédoine au début du siècle. En 1915, Lesbos vote pour la première fois depuis sa libération en 1912 et tous les députés de l'île sont favorables à 
Venizélos. Dans le même esprit, ses habitants ont soutenu en 1917 le mouvement politique et militaire de la "Défense nationale » et le gouvernement de Venizélos à Thessalonique. C'est dans ce cadre que Myrivilis, ainsi que tout un bataillon de Lesbos, se trouve le 8 mai 1917 à Monastiri (via Thessalonique) pour combattre les Bulgares et les Allemands aux côtés des Alliés, notamment les Français. D'ailleurs, les Grecs ont été entraînés, équipés et dirigés par les Français ${ }^{6}$. C'est à partir de cette époque que

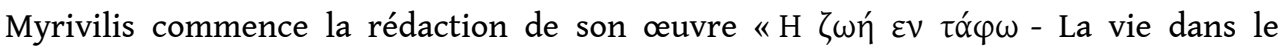
tombeau » qu'il a publiée pour la première fois en 42 épisodes, entre 1923 et 1924, dans le journal « Kambana » qu'il éditait lui-même. Avant son édition finale en 1955 par la maison « Estia ", elle a connu plusieurs éditions avec des changements et des rajouts".

4 La vie dans le tombeau sera non seulement le roman peut-être le plus important de Myrivilis, mais aussi une œuvre de référence dans les lettres grecques. Il prend pour nous une autre dimension et nous concerne directement puisqu'il se déroule en Macédoine. L'intérêt est double puisque la question traitée n'est pas un sujet imaginaire, vécu par des héros «fabriqués » dans un lieu irréel. Bien au contraire, le sujet est bien la Première Guerre mondiale, les héros - ou pour être plus précis - les personnages sont l'écrivain lui-même et ses compagnons, parmi lesquels son frère. Quant au lieu, il n'est rien d'autre que la Macédoine.

5 À noter que Myrivilis fait partie du courant littéraire que Beaton a appelé «école éolienne - école du témoignage ». L'œuvre de ces écrivains, tous originaires d'Asie Mineure et de l'île de Lesbos, n'est que l'expérience vécue : les conflits entre Grecs et Turcs, l'effondrement de l'Empire ottoman et les guerres successives qu'il a générées, ainsi que la Catastrophe d'Asie Mineure font partie du quotidien des écrivains comme Venezis, Doukas, Kontoglou, Sotiriou, Myrivilis.

6 Myrivilis décrira dans cette œuvre les expériences cauchemardesques et l'absurdité de la guerre qui transforme l'homme en animal, des expériences qui vont poursuivre l'écrivain tout au long de sa vie et qui vont hanter toute son œuvre. Ainsi La vie dans le tombeau deviendra l'expression de tous les idéaux antimilitaristes et constituera un roman précurseur pour son époque ${ }^{8}$. Cette œuvre dont la contradiction du titre "vie » et «tombeau ", est empruntée aux chants de la Passion du Christ le Vendredi saint, n'a pas comme but de fabriquer des héros, des surhommes qui maitrisent les événements et sortent toujours victorieux, mais au contraire de mettre en valeur l'antihéros, qu'il s'agisse du narrateur lui-même, de ceux qui combattent à ses côtés, ou bien de ses ennemis. C'est ainsi que l'œuvre est traversée d'un humanisme qui la rend unique dans son genre et pour son époque.

7 Le caractère autobiographique d'une part, et la volonté de garder l'objectivité de la narration des faits historiques d'autre part, font de l'œuvre un témoignage humain contre la cruauté et la mort. Sa langue, entre la démotique la plus pure et le langage journalistique, sait maintenir l'intérêt du lecteur qui se laisse entraîner à devenir luimême un témoin. Malgré les longues descriptions qui risquent parfois de fatiguer, le réalisme et la passion romantique rendent le texte très souple. Pour obtenir cette composition, Myrivilis suit deux axes principaux : en réalité il découpe son œuvre en une série de scènes-chapitres qui décrivent la guerre proprement dite et en une série qui parle de la paix. Les chapitres dont le thème est la paix sont placés lorsque l'ambiance devient trop lourde, permettant ainsi d'alléger la narration crue et douloureuse et de reposer le lecteur. Ils ponctuent l'ensemble du roman de façon à 
obtenir un équilibre parfait dans la structure de l'œuvre. C'est d'ailleurs grâce à cette alternance qui crée un effet de contraste que l'écrivain dénonce et décrit la guerre9 .

Pour notre travail, nous nous concentrerons sur un chapitre qui décrit la guerre et sur deux autres dont le contenu est constitué de descriptions pacifiques.

Avec le chapitre "la Cité Fantôme ", l'écrivain nous place directement au cœur de la guerre sur l'un des fronts les plus importants, juste en dehors de la ville de Monastiri. La traversée de Monastiri pour rejoindre le front tenu par les Français est une occasion pour Myrivilis de nous parler de cette ville bien qu'il n'y reste que quelques heures : il commence en effet son texte en notant qu'ils sont entrés et sortis de la ville durant la nuit. Pourtant, il prendra le temps de faire une esquisse rapide et significative, sur la cruauté de la guerre et les conditions de vie bien sûr, mais aussi sur la composition de la population, les sentiments des gens et leur situation durant les dernières années. La troisième phrase du premier paragraphe fait une allusion politique : «c'est une grande cité serbe dont les habitants sont des Grecs ». L'écrivain devient le témoin de cette réalité et exprime son étonnement par cette phrase courte et significative. Il fait allusion à la perte de la ville de Monastiri en 1912 pendant la Première Guerre balkanique. L'armée grecque s'était alors retrouvée face à deux options : continuer la route vers l'ouest ou se diriger vers Thessalonique. L'histoire est connue: Venizélos devant le danger d'abandonner Thessalonique aux Bulgares a ordonné à l'héritier Constantin, chef des armées, de laisser Monastiri aux Serbes. À peine cinq années plus tard après cette perte, ou plutôt cet abandon, à l'occasion de cette nouvelle guerre les Grecs se trouvent à nouveau dans cette ville face à une population grecque conséquente qui les attend pour se libérer! Myrivilis après une description rapide tel un compte rendu purement journalistique fait parler les habitants qui apparaissent petit à petit venant de nulle part. Après s'être assurés qu'il s'agit de soldats grecs, ils se livrent à des confessions et à des prières qui témoignent de leur espoir et qui mettent les soldats dans l'embarras. Par un dialogue très bref, dense et intense, l'écrivain fait immédiatement ressortir la complexité de la situation en Macédoine: «Etes-vous vraiment nos frères ? Êtes-vous Grecs? Grecs de Grèce ? " « Mais oui ». « Nous vous attendions depuis des années dans l'esclavage. Nous rêvions de vous, nous faisions de vous des chansons, nous vous vénérions sans même vous connaître... Ne nous laissez plus, nos frères, aux Serbes. Ils nous torturent durement parce que nous sommes des Grecs! » Et un vieillard ajoute : « ils nous battent avec le fouet quand ils nous entendent parler grec, quand nous célébrons la messe en grec. Ils nous ont pris les églises, nos belles écoles. Ils violent nos femmes... ». Myrivilis parle dans ce passage des méthodes violentes et radicales qui ont été employées pour changer les données de la composition de la population en la rendant serbe. Même si cette ville n'était pas exclusivement habitée par des Grecs, comme l'écrivain l'a souligné, elle était loin d'être une ville slave. Ce n'était donc pas une petite minorité, mais une partie significative de la région de la Macédoine centrale qui était concernée. Le prosélytisme plutôt pacifique d'avant les Guerres balkaniques envers la religion et la langue avait fait place à ces procédés plus violents ${ }^{10}$. Bien évidemment, le lecteur, mais avant tout le narrateur écrivain lui-même, subit un premier choc et il exprime sa confusion. La question n'est même pas de savoir comment ils pourront aider cette population opprimée, mais met en cause les raisons de leur intervention dans ce conflit : "Seigneur! Mais est-ce que nous sommes venus combattre les Serbes pour libérer les Grecs ou bien pour faire la guerre contre les Bulgares et les Allemands pour libérer nos alliés les Serbes que le roi a trahis?» Nous pouvons facilement comprendre le désarroi du romancier devant cette 
situation difficile sans précédent qui exprime parfaitement cette fameuse "salade macédonienne » où les frontières sont en permanente construction, où les guerres se succèdent et où les alliances se font et se défont au détriment des populations.

Nous pouvons ensuite étudier les chapitres « La maison de la bonté » et « Zavali Maïko Pauvre Mère » qui, bien qu'interrompus par un troisième forment à notre avis un ensemble. L'écrivain nous décrit son séjour en pleine campagne macédonienne dans une famille de villageois, près des rives de Dragoras en dehors de Monastiri. Le narrateur, blessé à la jambe pendant les combats, a été placé dans cette famille pour sa convalescence. C'est donc pour lui un moment de calme et de sérénité, une courte pause pendant des guerres acharnées et la vie inhumaine des tranchées sur le front. En conséquence, l'écrivain nous décrit avec enthousiasme l'accueil qu'il a reçu et ne manque pas de nous présenter l'habitat de ces gens, leur quotidien sans oublier de décrire leur physique et leurs traits de caractère. Il en profite pour mettre des images d'une vie pacifique rythmée par le travail de la terre et la joie d'un quotidien simple en contraste avec la vie des tranchées, démontrant ainsi une fois de plus l'absurdité de la guerre et de la mort. D'ailleurs des expressions comme "ambiance pacifique », «la maison de la paix ", "asile sacré » qu'on retrouve çà et là au cours du texte - à commencer par l'intitulé du chapitre lui-même «maison de la bonté »- ne font qu'accentuer ce contraste.

11 Dans ce même passage, Myrivilis pose également la question des origines de ces gens, rendant ainsi le texte très actuel, étant donné l'intérêt politique des dernières décennies. Nous allons donc nous intéresser au regard que l'écrivain porte à ces personnes, point de vue d'un soldat grec ou d'un journaliste présent sur le terrain en 1917, avant que la Macédoine ne finisse par être transformée en question de territoire national. Myrivilis, homme de lettres, est loin d'avoir un regard «de politicien». À travers des dialogues toujours brefs, mélangeant le discours direct et ses pensées personnelles, utilisant des mots tantôt grecs tantôt slaves, l'écrivain nous livre un témoignage précieux. Il nous donne une idée très précise sans vouloir récupérer la situation. C'est pourquoi quand la mère explique à ses deux petites filles qu'il s'agit d'un soldat grec, un "vrai chrétien ", "dobro kristian", écrit en utilisant des caractères grecs, il se tourne vers le lecteur pour dire qu'en réalité, «à ce moment-là précisément, il n'était ni chrétien, ni grec, ni serbe, mais tout simplement un homme plein de désirs, de nostalgie et de fatigue ${ }^{11}$. C'est après cette mise au point qu'il dira de ces gens qu'ils ne sont ni Bulgares, ni Serbes, ni Grecs.

L'appellation "macédonien » avait alors une connotation géographique qualifiant tous les habitants de cette région: les Grecs s'appelaient "Makedones", les Bulgares «Makedontsi » et les Valaques «Macedoneni ». C'est dans ce contexte que Gladstone a prononcé sa fameuse phrase «la Macédoine aux Macédoniens» qui concernait sans ambiguïté tous les groupes ethniques, d'origines et de religions diverses ${ }^{12}$. D'ailleurs selon Evangelos Kofos, l'adjectif «makedontsi » a été utilisé durant le XIX ${ }^{\mathrm{e}}$ siècle pour distinguer les Bulgares de Macédoine des Bulgares de la Bulgarie autonome et de ceux du vilayet d'Andrinople qui se qualifiaient de «Trakiitsi». Il s'agit donc d'une désignation géographique qui devient encore plus significative quand, toujours selon Kofos, ces Bulgares révolutionnaires ont manifesté ouvertement leur opposition à une annexion directe de la Macédoine à l'État bulgare alors qu'à peine quelques années plus tard, en 1912-13, ces "opposants » ont choisi de combattre dans le camp bulgare ${ }^{13}$. Il nous semble clair que nous ne pouvons pas parler d'une idéologie nationale nouvelle et 
déterminée. C'est d'ailleurs dans ce contexte que Myrivilis comprendra, lui aussi le terme "makedon" et l'utilisera par la suite sans aucune signification politique. D'ailleurs comme Peter Mackridge le note bien, en utilisant justement comme exemple le cas de Myrivilis, mais aussi l'œuvre de Pinelopi Delta et d'Ion Dragoumis, les Grecs ont utilisé jusqu'aux années 30 le terme «macédonien » pour qualifier le parler slave de cette région qui était désignée comme « Macédoine ${ }^{14}$.

Ainsi dans les premières éditions de son travail ${ }^{15}$, Myrivilis, en commentant les sentiments de cette famille à travers leur identité culturelle, l'écrivain note sans hésitation et ambiguïté que ces gens ne veulent être ni Bulgares, ni Serbes, ni Grecs, seulement "Makedon ortodoks ${ }^{16}$ écrit phonétiquement en caractères grecs. En revanche, quand, durant les années 30 et surtout après la Deuxième Guerre mondiale, le nom «Macédoine » et l'adjectif "macédonien» commencent à être utilisés politiquement par la Yougoslavie de Tito, Myrivilis enlève ce terme qui n'est plus compris de la même façon. Ainsi, dans l'édition définitive, cette famille ne veut toujours pas être Bulgare, ni Serbe, ni Grecque, mais nous ne savons rien de plus. Il est évident que l'écrivain ne pourra plus utiliser un terme géographique au départ, mais qui prête désormais à confusion.

Notons pour autant que ce changement n'est pas le seul ; comme nous l'avons déjà mentionné, le roman a eu plusieurs éditions avec de nombreuses modifications jusqu'à son édition définitive de 1955. Alors qu'il n'était au départ qu'une série d'articles de journal, il compte déjà en 1931 quelque deux cents pages qui deviendront par la suite quatre cents. À ajouter également qu'aucun autre changement concernant le parler, la religion ou l'identité culturelle en général, n'a été apporté pour modifier le sens. Bien au contraire, comme pour le livre dans son ensemble, ces passages ont été enrichis, les phrases retravaillées, renforçant même parfois la particularité de caractère du personnage principal - la mère - et à travers elle, de toute cette population de cette partie de la Macédoine. Signalons aussi que, durant toute la période de la dictature de Metaxas ainsi que pendant l'occupation, le livre était interdit de circulation bien que Myrivilis ait été considéré par la critique littéraire comme un sympathisant du régime ${ }^{17}$. Bien évidemment il faut être prudent et tenir compte du changement des opinions politiques de l'écrivain : même si Myrivilis n'a jamais adhéré ouvertement aux idées de Metaxas, il ne s'y est néanmoins jamais opposé ouvertement, malgré ses idées progressistes et sa position proche de Venizélos. Cependant, quelle que soit l'orientation politique de l'écrivain, ce qui nous semble intéressant c'est que, devant cette nouvelle utilisation du terme "Macédoine", désormais politique et non plus géographique, il réagit, exprime son désaccord et met son texte à jour.

Mais, pour revenir à notre sujet, la question de Macédoine n'a en réalité jamais été un conflit uniquement gréco-bulgare. Il convient de rappeler que, déjà après le congrès de Berlin de 1878 qui revoyait les accords de San Stefano, les Serbes - comme d'ailleurs les Roumains - sont entrés dans le « jeu » pour profiter de ces territoires qui échappaient à la Grande Bulgarie et qui restaient désormais en dehors de ses frontières ${ }^{18}$. C'est à ce moment-là que les Serbes se pressent de qualifier de "Serbes » les slavophones de la Macédoine dans le cadre d'une nouvelle politique nationale selon laquelle ces populations ne seraient pas des Bulgares, mais des Slaves. Myrivilis confirme tout cela dans son texte. Il explique que les enfants de sa famille d'accueil, de cette "mère courage ", faisaient la guerre aux côtés des Bulgares alors que juste avant ils étaient dans l'armée serbe ${ }^{19}$. Cette situation rappelle le drame du protagoniste de Dido Sotitiou 
dans Terres de sang ${ }^{20}$ et avec lui le sort de tous les Grecs d'Asie Mineure, qui ont fait la guerre en tant que Grecs avec l'armée grecque et en tant qu'Ottomans avec l'armée turque. Le cas est bien évidemment loin d'être exceptionnel, il reste néanmoins significatif. La narration devient dramatique, le ton change, le malaise exprimé par le romancier est aussitôt ressenti par le lecteur. Le passage nous rappelle l'ironie de la tragédie antique, le narrateur devient tout d'un coup le soldat grec qui combat les Bulgares. Par conséquent, les enfants de cette famille sont automatiquement ses ennemis ${ }^{21}$ et la mère soigne probablement le tueur de ses fils : « Pourtant, cela aurait pu parfaitement arriver une nuit, pendant des patrouilles qui frappent aveuglément, que le cœur de ses enfants se trouve face à ma baïonnette » nous dit-il, mais une fois de plus la paix triomphe et Myrivilis fait de cette femme un symbole : «Sa compassion tombe sur moi comme la pluie propre qui vient du ciel. Sans s'indigner, sans réserve amère, sans se plaindre."

Un autre sujet très intéressant qui vient compléter l'image que l'écrivain nous propose pour ces populations qui habitaient la partie centrale de la Macédoine, c'est leur langue ou pour reprendre le terme utilisé «leur idiome - $\gamma \lambda \omega \sigma \sigma$ có $1 \delta i ́ \omega \mu \alpha »^{22}$. Myrivilis accorde à cette langue une importance particulière, non seulement parce que la communication deviendra vitale et que la langue est l'outil par excellence pour connaître ce groupe humain et à travers lui tout ce monde, cette culture et la mentalité, mais aussi, car lui-même en tant qu'écrivain est sensible à la façon de parler des gens. Cette expérience très personnelle lui permettra également de noter que cette « langue est une branche slave avec beaucoup d'éléments turcs et grecs $»^{23}$ et que «les Serbes ainsi que les Bulgares la comprennent ${ }^{24}$. Il ne s'agit donc pas, selon Myrivilis, d'une langue formée au sens national du terme ou associée à un territoire déterminé. Les terminaisons des noms en -evski ou en -ovski qui ont remplacé celles en -ev ou en -ov, trop bulgares et celles en -its, trop serbes étaient, semble-t-il très éloignées des préoccupations du peuple à l'époque de Myrivilis ${ }^{25}$.

Il sera dès le premier contact séduit par la sonorité de cette langue dont le manque de

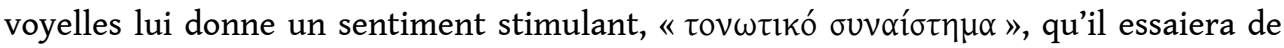
nous décrire avec des onomatopées ou avec des images comme l'exemple du vol d'oiseau ou des galets entraînés par le courant du Dragoras, la rivière qui traverse la région ${ }^{26}$. Il sera également fasciné par la confusion que provoque l'opposition du « oui » grec et du «non » slave, termes qui sonnent tous deux pareils ${ }^{27}$. Il mentionnera tout de suite qu'il tient un glossaire qu'il complète tous les jours ${ }^{28}$. Le lecteur sera désormais entraîné par le héros dans ce Nouveau Monde qu'il découvre comme les enfants découvrent le monde pour la première fois. La force de la langue dépasse les différences et rapproche les gens; au-delà d'un code utilitaire, elle ouvre le cœur, libère les sentiments des gens et enfin installe la confiance pour les unir. Des phrases comme



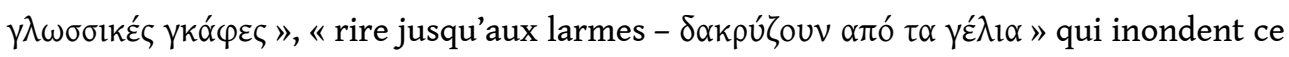
passage démontrent la volonté de Myrivilis de mettre en avant la spontanéité de l'âme et l'humanisme pour effacer les distances.

Une autre question que Myrivilis aborde concernant les habitants des rives du Dragoras, c'est la foi chrétienne et son rôle déterminant dans leurs relations avec l'ensemble de la population macédonienne. L'écrivain précise que les villageois n'aimaient pas les Serbes, car ils les traitaient de Bulgares, ils n'aimaient pas les Bulgares non plus, car ces derniers prenaient leurs enfants à la guerre ${ }^{29}$ puisque ceux-ci 
combattaient aux côtés des Bulgares lors de la Deuxième Guerre. Au contraire, leurs relations avec les Grecs semblaient être plus proches puisqu'ils éprouvaient, note le

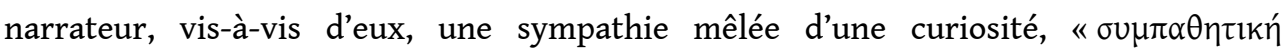
$\pi \varepsilon \rho \iota \varepsilon ́ p \gamma \varepsilon l \alpha$ », car les Grecs étaient les authentiques disciples du Patriarche œcuménique. Il ne manque pas l'occasion de parler de la fascination que Byzance continuait à exercer dans les Balkans en mentionnant qu'il y avait toujours leurs évangiles, leurs icônes et même les tombes des prêtres et de leurs notables écrits en grec. Certainement Myrivilis ressent cette relation à travers le christianisme orthodoxe et la notoriété de l'Église de Constantinople, car comme nous l'avons déjà mentionné, avant tout il n'est qu'un «orthodoxe, un vrai ». Il est intéressant de voir également comment l'objectivité de l'écrivain et son regard distant de journaliste - dirait-on - le pousse à saisir le moment pour critiquer le haut clergé et ses pratiques, pour la collecte des impôts. L'écrivain partisan d'une justice sociale est sensible aux abus du pouvoir politique ou ecclésiastique $^{30}$. Le texte semble très proche de la réalité du moment, puisqu'il explique parfaitement comment les habitants sont pris au piège de la rivalité gréco-bulgare entre le Patriarcat œcuménique et l'Église indépendantiste bulgare, l'Exarchat. Pour citer Kofos, ils avaient le choix entre rester fidèles à Constantinople et être considérés comme des Grecs ou bien fidèles à l'Exarchat et être pris pour des Bulgares ${ }^{31}$. Naturellement Myrivilis semble ignorer les théories qui ont été développées - toujours en citant Kofos - par les historiens yougoslaves et selon lesquelles une élite locale s'était organisée pour créer une idéologie dite "macédonienne " pour rassembler les slavophones en considérant les Guerres balkaniques, non plus comme le conflit armé entre Grecs, Serbes et Bulgares dans l'espace macédonien occupé par les Turcs, mais comme le combat des "Macédoniens » contre " les idéologies nationales étrangères " 2 . À noter également que l'expérience de l'écrivain ne concerne pas les populations musulmanes, souvent albanophones qui pendant l'occupation ottomane étaient assimilées automatiquement aux Turcs et qui seront considérées comme «macédoniennes » seulement durant la période yougoslave pour freiner l'albanisation de la population ${ }^{33}$.

19 Selon Myrivilis dans ce roman, même au début du XXe siècle quand les rivalités culturelles sont devenues des combats armés qui opposaient les différents peuples (Grecs contre Bulgares, Bulgares contre Serbes, Valaques roumanophones contre Grecs), la question d'une idéologie nationale dans l'espace macédonien ne se pose aucunement. Les gens s'allient selon leurs besoins avec les Serbes ou les Bulgares, tandis qu'en même temps ils se sentent proches des Grecs et éprouvent une sympathie à leur égard. Cette sympathie est due à l'héritage orthodoxe commun. Ils parlent une langue difficile à déterminer, au moins pour Myrivilis, entre le bulgare et le serbe avec des éléments turcs et grecs. Il est vrai que les efforts du XIX pour former une conscience de nation se sont montrés vains. La question de la survie a été le souci premier surtout pour tous ces gens qui habitent la partie centrale de la Macédoine qui restera la plus hétérogène du point de vue ethnologique. L'appartenance à une communauté avec des liens culturels et religieux ayant une langue commune est le point de contact entre les citoyens et l'État «national »" Néanmoins pour qu'une nation soit considérée comme telle et pour qu'elle puisse former un État, elle doit avoir une conscience politique commune, sinon l'unité culturelle seule ne peut concerner qu'une ethnie ${ }^{35}$.

20 Avec cette œuvre Myrivilis réussit certainement une composition sans précédent pour décrier les catastrophes de la guerre et la vanité humaine. Son style très critique qui 
touche à la satire et à la caricature est dominé par un lyrisme qui ne connaît pas de

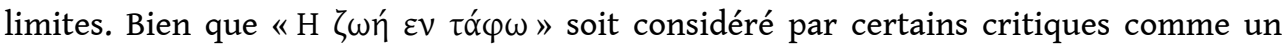
rassemblement de chapitres fragmentaires ${ }^{36}$, il réussit un équilibre parfait entre la guerre et la paix grâce à une maîtrise de la langue au registre très varié qui lui permet d'exprimer les sentiments les plus divers ${ }^{37}$ et d'obtenir une immédiateté entre narrateur, héros et lecteur. Ainsi Myrivilis échappe - selon la phrase de Mario Vitti - au naturalisme expressionniste et à son héritage du XIXe siècle. Il devient précurseur allant au-delà de la génération des années 20 à laquelle, par son âge, il appartenait de fait. En reproduisant de façon convaincante le style naïf de la narration populaire, il peint une série d'antihéros à l'opposé de la tragédie grecque ancienne et du « surhomme » nietzschéen ${ }^{38}$ qu'il a créé plus tard avec Notre-Dame la Sirène et avec Vassilis l'Albanais.

21 En même temps il obtient un témoignage précieux sur la Macédoine de la période de la Première Guerre mondiale qui nous permet d'avoir une idée précise et claire sur le regard que son époque lui porte. Myrivilis, malgré lui, nous permet de revoir certaines pages de cette histoire à travers sa propre expérience ; ses contacts directs et profonds avec les gens, même s'ils sont parfois idéalisés, restent sincères et honnêtes puisque son premier souci est de désavouer la guerre et de ne faire ni de l'histoire ni de la politique.

Avec des images des Grecs de Monastir pris au piège du nationalisme serbe et des villageois slaves qui ne sont ni Serbes ni Bulgares, mais qui font la guerre tantôt aux côtés des uns tantôt aux côtés des autres, l'écrivain ne peut que reconnaître l'existence d'un problème sérieux au sein de cette région, mais il est loin de considérer tel ou tel peuple comme héritier exclusif de l'histoire macédonienne. Le fait que l'utilisation des noms et des adjectifs concernant la Macédoine soit dépourvue de toute connotation politique et encore moins nationale ou nationaliste, mais purement géographique, laisse entendre que Myrivilis en tant qu'intellectuel de l'époque et militant engagé qui a même fait les guerres de 1912-1913 avant de participer à la Première Guerre mondiale, n'a jamais considéré cette région comme un espace fermé réservé à une seule et unique ethnie ou nation. De même les commentaires sur le parler des populations, les observations à propos des mots et surtout les remarques quant aux contacts avec les autres langues excluent selon Myrivilis toute idée d'une langue à part entière et autonome.

L'écrivain observe un monde complexe et fragile en devenir, reconnaît des problèmes, mais il garde toujours sa naïveté et met en avant l'humanisme pour surmonter les différences et vivre ensemble en paix. La mère de son ennemi le soigne sans amertume et devient le symbole du dépassement des frontières. Le narrateur à son tour répond avec sa volonté de parler sa langue, d'aller vers elle et de partager la même culture.

BIBLIOGRAPHIE

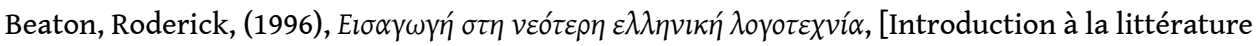
grecque moderne], Athènes, Nefeli. 


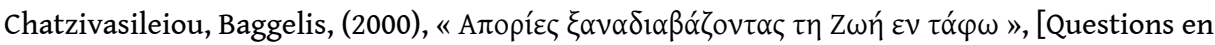
relisant le roman « La vie dans le tombeau »], Nea Estia, $n^{\circ} 1725$.

Dimadis, Konstantinos, (2004), $\Delta \imath \kappa \tau \alpha \tau o \rho i ́ \alpha, ~ \Pi o ́ \lambda \varepsilon \mu o \zeta ~ \kappa \alpha l ~ \Pi \varepsilon \zeta o \gamma \rho \alpha \varphi i ́ \alpha$ 1936-1944, [Dictature, Guerre et Prose 1936-1944], Athènes, Estia.

Georgeon, François, (1989), « Le dernier sursaut 1878 - 1908 », in Histoire de l'Empire Ottoman, Paris, Fayard.

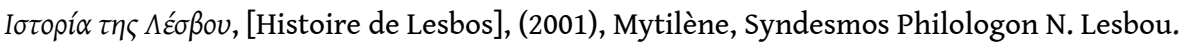




première à la Deuxième Guerre mondiale], Athènes, Sokolis.

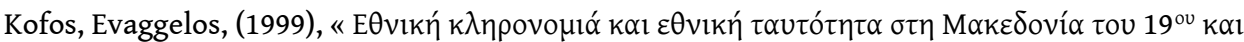

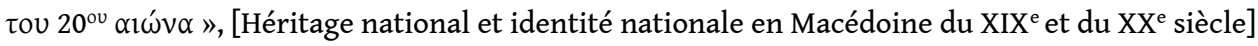

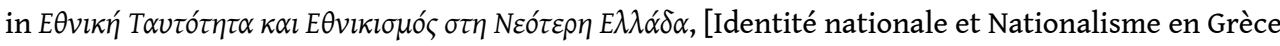
Moderne], Athènes, MIET.

Mackridge, Peter, (2009), Language and national identity in Greece, 1766-1976, [Langue et identité nationale en Grèce, 1766-1976], Oxford: Univ.Press.

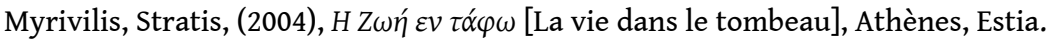

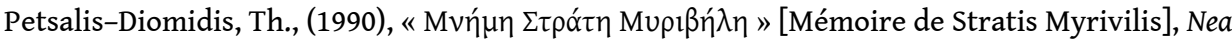
Estia $\mathrm{n}^{\circ} 1523$.

Sotiriou, Dido, (1983), M $\alpha \tau \omega \mu \varepsilon ́ v \alpha \chi \omega ́ \mu \alpha \tau \alpha$, [Terres de sang], Athènes, Kedros.

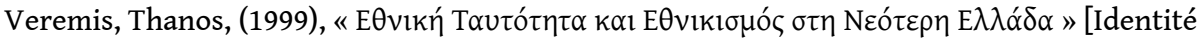

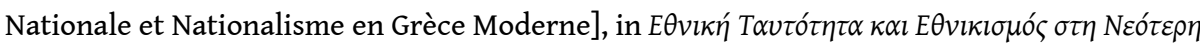
$E \lambda \lambda \alpha ́ \delta \alpha$ [Identité Nationale et Nationalisme en Grèce Moderne], Athènes, MIET.

Vitti, Mario, (1989), Histoire de la Littérature grecque moderne, Paris, Hatier.

\section{NOTES}

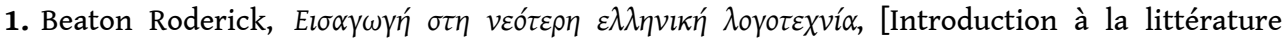
grecque moderne], Athènes, Nefeli, 1996, pp. 120-9.





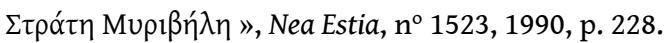

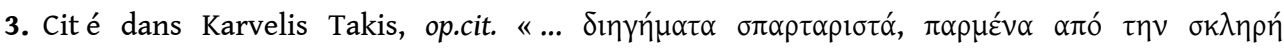

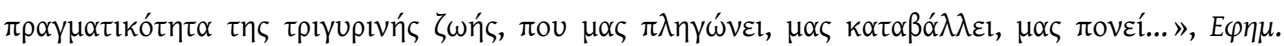

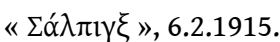

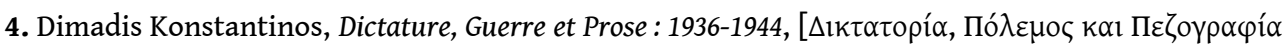
1936-1944], Athènes, Estia, 2004, pp. 168, 180, 183, 188, et Vitti Mario, Histoire de la Littérature Grecque Moderne, Athènes, Hatier, 1989, pp. 366-7.

5. Karvelis Takis, op.cit, p. 103.

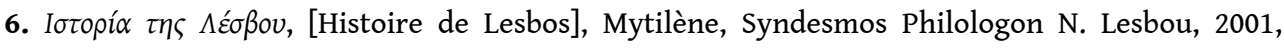
pp. 245-252.

7. Karvelis Takis, op.cit, pp. 98-99.

8. Ibidem, p. 105. 


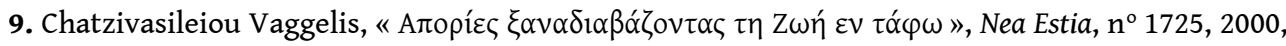
p. 96.

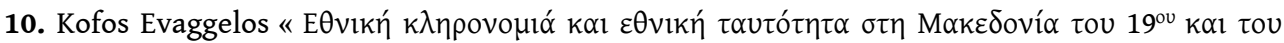
$20^{\circ v} \alpha \iota \omega ́ v \alpha$ » in Identité Nationale et Nationalisme en Grèce Moderne, [E $\theta v ı$ เ

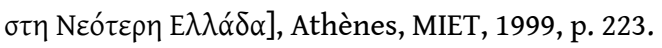

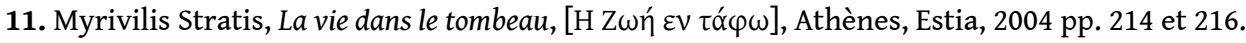

12. Kofos Evaggelos, op.cit., pp. 215, 216.

13. Ibidem, p. 216.

14. Mackridge Peter, Language and national identity in Greece, 1766 - 1976, Oxford, University Press, 2009 , p. 303.

15. Notamment à celle de 1931 que nous avons également consultée.

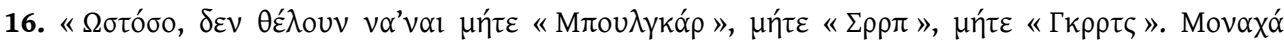

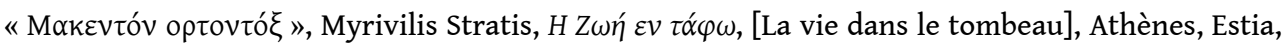
1931, pp. 104-105.

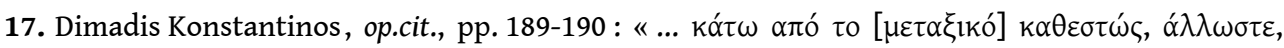

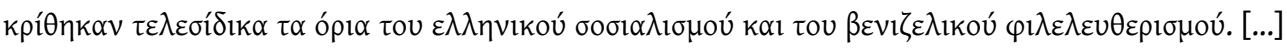

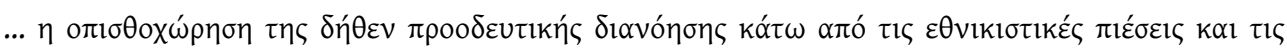

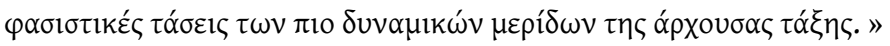

18. Georgeon François, «Le dernier sursaut 1878 - 1908 » in Histoire de l'Empire ottoman (Mantran Robert ed.), Paris, Fayard, 1989, p. 559.

19. Myrivilis Stratis, op.cit., p. 229.

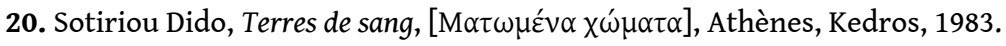

21. Myrivilis Stratis, op.cit., pp. 228-229.

22. Ibidem, p. 225.

23. Idem.

24. Myrivilis Stratis, op.cit., p. 227.

25. Kofos Evaggelos, op.cit., p. 226.

26. Myrivilis Stratis, op.cit., p. 225.

27. Ibidem, p. 226.

28. Ibid., p. 225.

29. Ibid., p. 227.

30. Myrivilis Stratis, op.cit., pp. 227-228.

31. Kofos Evaggelos, op.cit., p. 233.

32. Ibidem, pp. 233-234.

33. Ibid., p. 239.

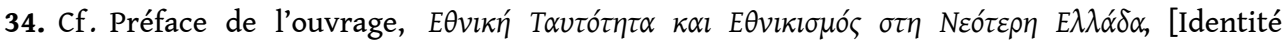
Nationale et Nationalisme en Grèce Moderne], rédigée par Veremis Thanos, Athènes, MIET, 1999, p. 27.

35. Idem.

36. Beaton Roderick, op.cit., p. 186.

37. Vitti Mario, op.cit., p. 327.

38. Beaton Roderick, op.cit., p. 229. 


\section{RÉSUMÉS}

L'écrivain Stratis Myrivilis traduit son expérience de la guerre en Macédoine, son désarroi en découvrant l'identité culturelle "macédoniens orthodoxes".

Cet article examine la relation de Stratis Myrivilis, écrivain grec né à Lesbos en 1890 et mort à Athènes en 1967, avec la Macédoine, qu'il a connue en tant que soldat pendant les Guerres balkaniques de 1912-1913 puis lors de la Première Guerre mondiale de 1917 à 1919, au travers de son roman La vie dans le tombeau publié de 1923 à 1924 et plusieurs fois réédité. L'article souligne le désarroi du narrateur qui s'exprime dans le chapitre "La Cité Fantôme ", alors qu'il participe, en 1917, à la jonction des Grecs avec le front français, face à la complexité de la situation de la Macédoine où les frontières sont en permanente construction, où les guerres se succèdent et où les alliances se font et se défont au détriment des populations. D'une part il montre comment Myrivilis, dans les chapitres «La maison de la bonté » et "Zavali Maïko Pauvre Mère ", commente les sentiments des membres de la famille où, blessé, il passe sa convalescence, comment il analyse l'identité culturelle de ces gens qui ne se veulent ni Bulgares, ni Serbes, ni Grecs, mais seulement Macédoniens orthodoxes et enfin comment les habitants sont pris au piège de la rivalité gréco-bulgare entre le patriarcat đEcuménique et l'Église indépendantiste bulgare, l'Exarchat. D'autre part, il présente la position de l'écrivain qui semble prendre des distances quand le terme «Macédoine » est utilisé, par les voisins du nord, pour exprimer une entité politique et non géographique. En conclusion, l'article souligne l'humanisme de Myrivilis qui décrit les catastrophes de la guerre et la vanité humaine et prône la nécessité de surmonter les différences et de vivre ensemble en paix.

This article examines the relationship of Stratos myrivilis, -a Greek writer, who was born on Lesbos in 1890 and died in Athens in 1967- with Macedonia, which he knew as a soldier during the Balkan Wars of 1912-1913, and then during the First World War from 1917-1919. It is based on myrivilis's novel Life in the Tomb, which was published in 1923-1924 and reprinted several times. The article underscores the narrator's distress as expressed in the chapter "The Ghost Town", when he participated, in 1919,in the clash between the Greeks and the French, in the face of the complexity of the situation in Macedonia when the borders are constantly in flux, where one war follows another, and where the alliances are made and unmade at the population's expense. On the one hand it shows how Myrivilis, in the chapters "In the House of Kindnes" and "Zavali Maïko -Poor Mother" comments on the feelings of the members of the family where, wounded, he spends his convalescence, how he analyses the cultural identity of these people who regard themselves as neither Bulgarians, Serbs, nor Greeks but simply Macedonian Orthodox, and finally how the inhabitants fall into the trap of the Greco-Bulgarian rivalry between the Ecumenical Patriarchate and the independent Bulgarian Church, the Exarchate. On the other hand, it presents the stance of the writer, who seems to distance himself from the northern neighbours when they use the term "Macedonia" to describe a political entity rather than a geographical one. In conclusion, the article underlines the humanism of Myrivilis, who described the catastrophies of war and human vanity and advocated the need to surmount their differences and live together in peace. 
INDEX

Index géographique : Macédoine, Lesbos, Monastir

motsclesmk МАКЕДОНИЈА, БИТОЛА, ЛЕЗБОС

Mots-clés : Berlin (congrès de), Défense Nationale, identité macédonienne, Myrivilis Stratis

(1890-1969), Exarchat, propagande nationaliste, Myrivilis Stratis (1890-1969), pacifisme,

littérature grecque

Thèmes : Histoire, Littérature

Index chronologique : guerres balkaniques (1912-1913), guerre mondiale (1914-1918)

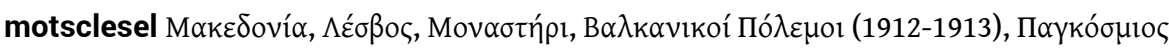



motsclestr Balkan Savaşları (1912-1913), Bitola, Dünya savaşı (1914-1918)

Keywords : macedonian identity, war novels, nationalistic propaganda, pacifism, greek-

bulgarian conflict, Macedonia, Lesbos, Monastir, Balkan Wars (1912-1913), History, Literature

\section{AUTEUR}

\section{GEORGES KOSTAKIOTIS}

CC. INALCO, Doctorant CREE-CEB EA4513 